\title{
Ion acceleration by multiple reflections at Martian bow shock
}

\author{
M. Yamauchi ${ }^{1}$, Y. Futaana ${ }^{1}$, A. Fedorov ${ }^{2}$, R. A. Frahm ${ }^{3}$, E. Dubinin ${ }^{4}$, R. Lundin ${ }^{1}$, \\ J.-A. Sauvaud ${ }^{2}$, J. D. Winningham ${ }^{3}$, S. Barabash ${ }^{1}$, and M. Holmström ${ }^{1}$ \\ ${ }^{1}$ Swedish Institute of Space Physics, Box 812, S-98128 Kiruna, Sweden \\ ${ }^{2}$ Centre d'Etude Spatiale des Rayonnements, Université de Toulouse, F-31038 Toulouse, France \\ ${ }^{3}$ Southwest Research Institute, San Antonio, TX 78228-0510, USA \\ ${ }^{4}$ Max-Planck-Institut für Sonnensystemforschung, D-37191 Katlenburg-Lindau, Germany
}

(Received February 4, 2011; Revised May 27, 2011; Accepted July 12, 2011; Online published March 8, 2012)

\begin{abstract}
The ion mass analyzer (IMA) on board Mars Express revealed bundled structures of ions in the energy domain within a distance of a proton gyroradius from the Martian bow shock. Seven prominent traversals during 2005 were examined when the energy-bunched structure was observed together with pick-up ions of exospheric origin, the latter of which is used to determine the local magnetic field orientation from its circular trajectory in velocity space. These seven traversals include different bow shock configurations: (a) quasi-perpendicular shock with its specular direction of the solar wind more perpendicular to the magnetic field (QT), (b) quasi-perpendicular shock with its specular reflection direction of the solar wind more along the magnetic field (FS), and (c) quasi-parallel (QL) shock. In all seven cases, the velocity components of the energy-bunched structure are consistent with multiple specular reflections of the solar wind at the bow shock up to at least two reflections. The accelerated solar wind ions after two specular reflections have large parallel components with respect to the magnetic field for both QL cases whereas the field-aligned speed is much smaller than the perpendicular speed for all QT cases. Key words: Mars, bow shock, acceleration.
\end{abstract}

\section{Introduction}

Collisionless shocks such as interplanetary shocks (Gloeckler et al., 1994) and bow shocks of planets, e.g., Earth (Paschmann et al., 1981; Sckopke et al., 1983, 1990) and Mars (Afonin et al., 1989; McKenna-Lawlor et al., 1998; Dubinin et al., 2006; Yamauchi et al., 2011) are known to accelerate ions. The accelerated ions at the terrestrial bow shock are observed as both diffuse distributions and discrete distributions in the energy domain (Bale et al., 2005; Burgess et al., 2005; Eastwood et al., 2005). An example of the diffuse distribution is the foreshock diffuse component (Gosling et al., 1978), and it has been explained mainly by Fermi acceleration. Examples of the discrete distribution are field-aligned beams and reflected gyrating ions (Gosling et al., 1978, 1982; Sckopke et al., 1983, 1990), and they have been explained by reflection at the bow shock (e.g., Burgess and Schwartz, 1984; Oka et al., 2005) and wave-particle interactions (e.g., Meziane et al., 2001; Mazelle et al., 2003), although the exact mechanisms are open questions. One consequence of reflection modeling is that some ions may go through multiple reflections or surfing (Sagdeev, 1966) to reach high energies. This model is further developed for thin shock case (Lee et al., 1996; Zank et al., 1996). The multiple reflections can also be the precondition for escape from the bow shock to form the foreshock (e.g., Oka et al., 2005), although multiple reflections

Copyright (c) The Society of Geomagnetism and Earth, Planetary and Space Sciences (SGEPSS); The Seismological Society of Japan; The Volcanological Society of Japan; The Geodetic Society of Japan; The Japanese Society for Planetary Sciences; TERRAPUB.

doi:10.5047/eps.2011.07.007 have not been observed for the terrestrial bow shock.

Recently, the Mars Express (MEX) Ion Mass Analyzer (IMA) has detected accelerated ions at multiple discrete energies (at least three energies) above the solar wind energy at locations within a proton gyroradius from the bow shock. Dubinin et al. (2006) showed an example of an energy-bunched structure in the spectrogram at two distinct energies in the foot region of the bow shock for a quasiperpendicular shock. The low energy branch of this structure is pick-up ions of newly ionized hydrogen in the exosphere. Yamauchi et al. (2011) analyzed an example of such an energy-bunched structure as summarized in Fig. 1. The spacecraft trajectory during this example is drawn in Fig. 2 (as \#7), in which the Mars-Sun Orbit (MSO) Cartesian coordinate system is used; i.e., the $+X$ direction points toward the Sun, the $+Z$ direction points northward in the ecliptic hemisphere, the $+Y$ direction completes the righthand Cartesian coordinate (i.e., pointing duskward), and $R^{2}=Y^{2}+Z^{2}$.

Figure 1(a) shows energy-time spectrograms of electron energy flux and proton energy flux observed by the ELectron Spectrometer (ELS) and IMA, respectively, during an outbound traversal from the frank bow shock to the upstream region on 12 July, 2005. The bow shock crossing is at around 11:34:30 UT. Solar wind protons are seen at around $0.7 \mathrm{keV}\left(\mathrm{He}^{++}\right.$contamination is seen at around $1.4 \mathrm{keV}$ ) every $192 \mathrm{sec}$ that corresponds to a scanning cycle of the field-of-view of IMA. Above the solar wind energy, energy-bunched stripes are observed at around $2 \mathrm{keV}$, $3 \mathrm{keV}$, and $5 \mathrm{keV}$. The lowest-energy branch is pick-up ions of exospheric origin (Dubinin et al., 2006; Yamauchi 


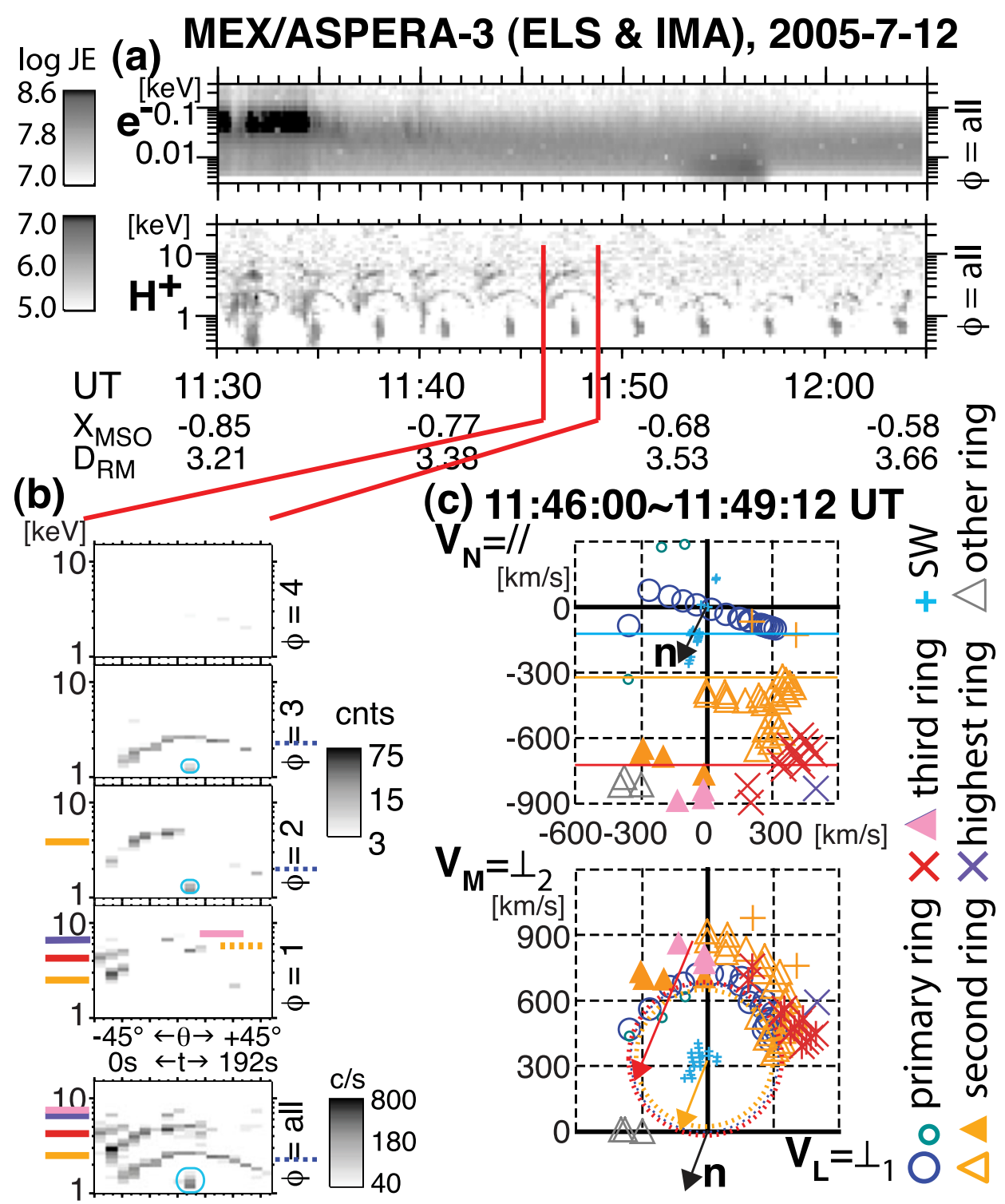

Fig. 1. Extractions from Yamauchi et al. (2011). (a) Energy-time spectrograms of the electron energy flux and ion energy flux (keV $\mathrm{cm}^{-2} \mathrm{~s}^{-1} \mathrm{keV}^{-1}$ ) observed by ELS and IMA, respectively, during 11:30-12:05 UT, 12 July 2005. Both spectrograms show the average of all 16 azimuthal directions. The nearly $3-\min (192 \mathrm{sec})$ cycle seen in the proton data is due to the scanning cycle of the IMA entrance (elevation) direction from $\theta=0\left(-45^{\circ}\right)$ to $\theta=15\left(+45^{\circ}\right)$. Solar wind protons are seen at around $0.7 \mathrm{keV}$ (contaminated by $\mathrm{He}^{++}$at around $1.4 \mathrm{keV}$ ) every $192 \mathrm{sec}$ scanning cycle. $D_{\mathrm{RM}}$ denotes the Mars-centric distance with a unit of Martian raduis. (b) The proton data during 11:46:00-11:49:12 UT for azimuthal sectors $\phi=1 \sim 4$ (counts per every $100 \mathrm{~ms}$ measurement), and integration of all sectors (counts per second). The first pixels correspond to $-45^{\circ}$ elevation $(\theta=0)$ and the last pixels to $+45^{\circ}$ elevation $(\theta=15)$. Different branches of the multiple stripes are marked by horizontal bars with different colors. (c) Velocity scatter plots in the $V_{L}-V_{N}$ projection (upper panel) and in the $V_{L}-V_{M}$ projection (lower panel) during the same scan as (b), i.e., 11:46:00-11:49:12 UT. The local Cartesian coordinates L-M-N are derived such that the lowest-energy ring distribution at around $2 \mathrm{keV}$, marked by blue dashed line in (b) and blue empty circles in (c), forms a gyrating circular trajectory (blue dashed circle in (c)) in the $V_{L}-V_{M}$ projection with zero average $V_{N}$, such that $\mathbf{N}$ points along the magnetic field (Yamauchi et al., 2011). The velocity space points with 5 counts or more during the $100 \mathrm{~ms}$ measurement period (this threshold is increased to 10 counts per $100 \mathrm{~ms}$ measurement for the solar wind that is marked by light blue pluses) are plotted in the spacecraft reference frame except for $\phi=0$ which is strongly contaminated from the other sectors. Identified ion populations in (b) are marked by different symbols (legend at right) using the same colors as (b). The modeled bow shock normal direction (n) is given by black arrows. The orange and red arrows show in the $V_{L}-V_{M}$ projection of the calculated jumps by the first and second specular reflections, starting from the solar wind velocity for the first reflection (orange) and from the end point of the observed second ring (corresponds to second injection) for the second reflection. Gyration trajectories of these reflected ions are given by orange and red dashed circles. The horizontal blue, orange, and red lines in the $V_{L}-V_{N}$ projection indicates the calculated field-aligned velocities $\left(V_{N}\right)$ of the solar wind, first reflected ions, and second reflected ions, respectively.

et al., 2006). Figure 1(b) shows ion data for azimuthal directions $\left(22.5^{\circ}\right.$ wide each) that registered these energybunched stripes during one of 192-sec cycles of the ion observation. The energy-bunched stripes at energies higher than that of the pick-up ions are marked by orange (around $3 \mathrm{keV}$ ) and red (around $5 \mathrm{keV}$ ). Signatures of the other pos- sible branches (around 6 8 keV) are also marked using different colors. Figure 1(c) shows the velocity scatter plot of protons for the same data using the same colors as Fig. 1(b), projected into planes along and perpendicular with respect to the estimated magnetic field direction $(-\mathbf{N})$, respectively. By taking the normal direction $\mathbf{n}$ of the empirical bow 


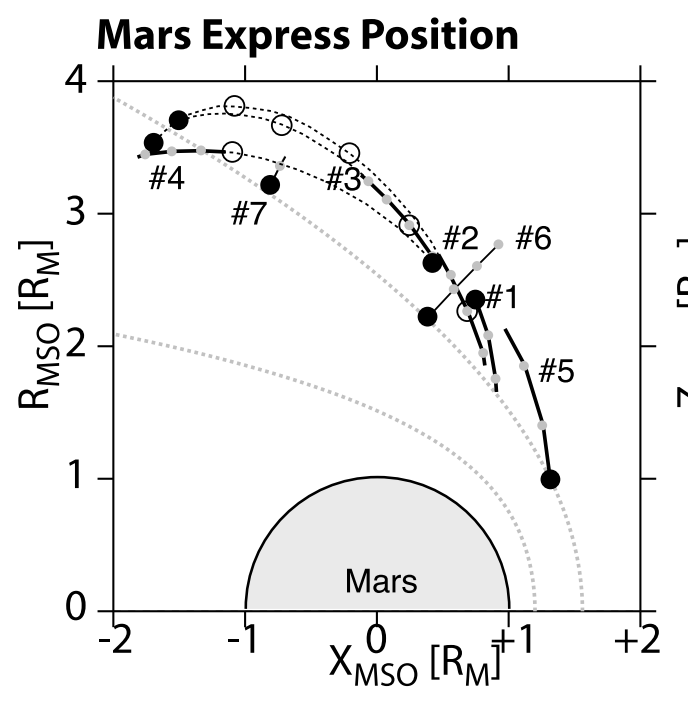

\#1: 2005-7-29, 17:26 17:49

\#2: 2005-8-03, 08:46 11:59

\#3: 2005-8-05, 08:22 10:35

\#4: 2005-11-22, 16:57 18:29

\#5: 2005-11-29, 16:05 16:33

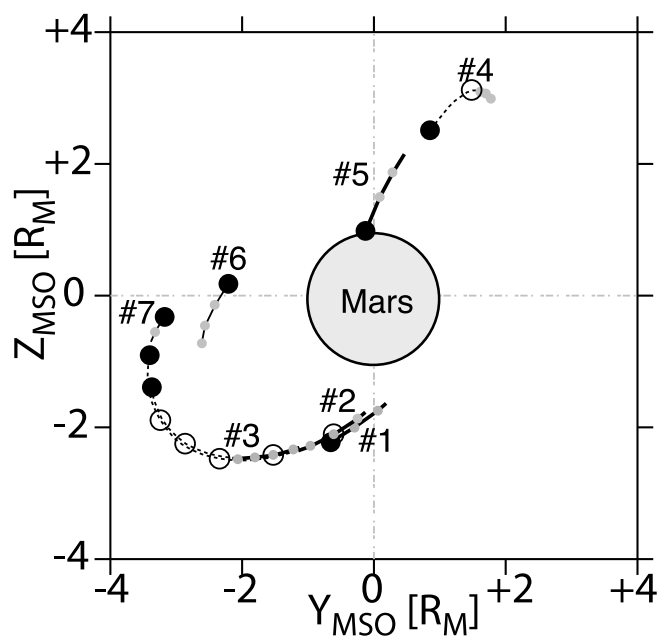

\#6: 2005-6-03, 05:59 06:29

\#7: 2005-7-12, 11:35 11:50

- Start of IMA observation or bow shock crossing

1 hour ticks $\quad 10$ min ticks

Fig. 2. Overview of the MEX orbits during seven traversals given in Table 1. The traversal when the multiple ring-like distribution was observed is drawn by using solid lines with grey circle marks every 10 min and otherwise using dashed lines with white circle marks every 1 hour. The dashed grey lines indicate the average position of the bow shock and magnetopause (Vignes et al., 2000). The unit $R_{\mathrm{M}}$ is the Mars radius. Cylindrical $R_{\mathrm{MSO}}=\sqrt{Y_{\mathrm{MSO}}^{2}+Z_{\mathrm{MSO}}^{2}}$ is used in the top left panel. The orbit numbers (\#1 to \#7) are the same as those in Tables 1-3.

shock model (Vignes et al., 2000), one can calculate the direction of a specular reflection from the assumed injection direction as:

$$
\mathbf{V}_{\text {ref }}-\mathbf{V}_{\text {in }}=-2\left(\mathbf{V}_{\text {in }} \cdot \mathbf{n}\right) \mathbf{n}
$$

where $\mathbf{V}_{\text {in }}$ and $\mathbf{V}_{\text {ref }}$ are velocities of injecting ions and specularly reflected ions. Since the value of (1) is not conserved over the Lorenz transform, Yamauchi et al. (2011) assumed the specular reflection in the Mars rest frame. The orange arrow in Fig. 1(c) (lower panel) shows the 2-D projection (onto perpendicular plane to the estimated magnetic field) of the specular reflection. The start and end of the arrows, respectively, correspond to the velocity space points of the solar wind (blue cross in Fig. 3(b)) and the reflected solar wind. The reflected ions are expected to gyrate in the solar wind frame, i.e., along the orange dashed circle. The horizontal blue and orange lines in Fig. 1(c) (upper panel) indicate the $V_{N}$ component (along the estimated magnetic field) of the injecting solar wind and specularly reflected solar wind, respectively. Both the orange dashed circle and orange line are consistent with the velocities of the observed second ring (orange empty triangle).

In Yamauchi et al. (2011), the same calculation was made for the second reflection because these reflected solar wind ions are expected to return back to the bow shock. If the bow shock is laminar, the incident angle of these returning ions to the bow shock will be very narrow, and hence the reflection can again be collimated to a narrow direction. The expected jump and trajectory are given by red arrow, red dashed circle, and red horizontal line, similar to the first reflection (orange color). They are consistent with the third ring distribution (red cross and pink triangle) which is collimated to a small velocity space. Such a collimation cannot be achieved by pick-up ions because they have wide phase angles for injection when they are reflected at the bow shock. Also, the intensity of the third ring cannot be explained by the reflection of the pick up ions. Therefore, Yamauchi et al. (2011) concluded that two main branches at energies higher than that of the pick-up ions represent a single and double specular reflections of solar wind protons at the bow shock, respectively. The specular reflection model agrees only in the Mars frame and not in the solar wind frame or de Hoffman-Teller frame that are sometimes used (e.g., Burgess and Schwartz, 1984).

As the possible cause for the multiple reflection specific to Mars but not reported at the Earth or Venus, Yamauchi et al. (2011) considered possible factors that makes Mars qualitatively different from Venus or Earth, namely the finite gyroradius effect compared to the radius of the bow shock curvature and the abundance of residual cold ions of exospheric origin near the Martian bow shock (Dubinin et $a l ., 1993)$. They demonstrated that the finite gyroradius effect makes the reflected ion easier to escape, i.e., more difficult to return back to the bow shock, whereas the cold ions near the Martian bow shock forms a third plasma population (others are the solar wind and the magnetosheath ions), making it possible to have a new "characteristic speed" which enables the double-foot structure as observed, and making the returning of the reflected ions easier. If this 
(a) QP shock \& gyration in the same plane (QT)

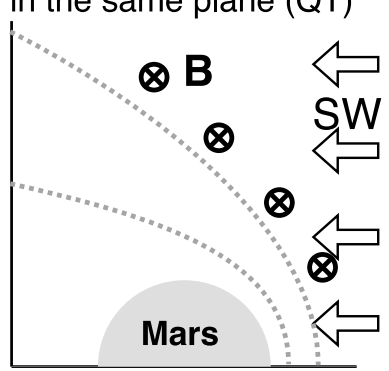

2005-7-29 (\#1)

2005-8-3, 11:46 UT (\#2a)

2005-11-22 (\#4)

2005-11-29 (\#5)

2005-6-3, 06:06 UT (\#6a) (b) QP shock \& gyration
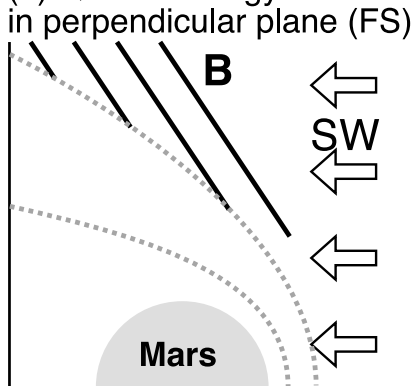

2005-8-3, 11:53 UT (\#2b)

2005-7-12 (\#7) (c) QL shock

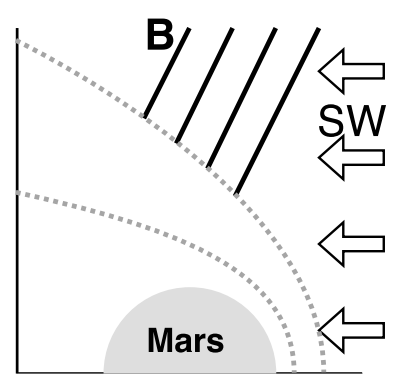

2005-8-5 (\#3)

2005-6-3, 06:13 UT (\#6b) (d) relation between QT, FS, and QL

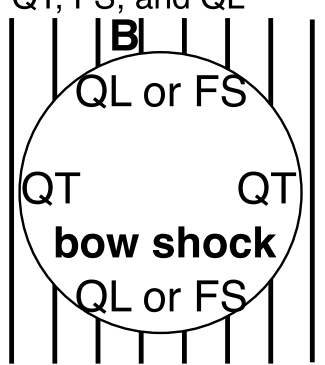

Fig. 3. Illustration of three different magnetic field configurations at the bow shock. The dashed grey lines indicate the average position of the bow shock and magnetopause (Vignes et al., 2000). Solid lines outside the bow shock (marked by B) denote the magnetic field orientation, and empty arrows to the right (marked by SW) denote the solar wind. (a) Quasi-perpendicular shock by which specular reflection direction is nearly perpendicular with respect to the magnetic field (QT). (b) Quasi-perpendicular shock by which specular reflection direction is oblique or parallel to the magnetic field (foreshock configuration, FS). (c) Quasi-parallel shock (QL). (d) The rightmost panel illustrates the azimuthal locations of three types of the shock when the bow shock is viewed from the Sun.

is true, we expect the multiple reflections at the Martian bow shock occur very often. So far, Yamauchi et al. (2011) showed only one example of quantitative examination due to the difficulty in deriving the magnetic field orientation. One example is not enough to draw a general conclusion. Furthermore, bow shock accelerations are different between quasi-perpendicular shocks and quasi-parallel shocks (e.g., Bale et al., 2005; Burgess et al., 2005). Therefore, more case studies with the same quantitative level are needed.

This type of analysis normally requires a statistical study; however, the Martian data existing to date are not suitable for statistical study because MEX does not carry a magnetometer for obtaining pitch-angles of observed ions even though MEX is the only spacecraft that produced a sufficient quantity of ion data at the relevant energies near the Martian bow shock for a statistical study. Although the local magnetic field orientation can sometimes be estimated from pick-up ions of exospheric origin, this method is not suitable for statistical studies when other ions (accelerated ions in the present case) overlay the pick-up ions (Yamauchi et al., 2011). In this paper, we examined the best cases from one-year of data (during 2005), i.e., when the energybunched structure is observed together with pick-up ions from the newly ionized hydrogen corona with a good separation in velocity space.

Here, we subdivide the observations into three categories in terms of shock types and the expected pitch angle of the specularly reflected solar wind in the Mars frame as illustrated in Fig. 3: (a) quasi-perpendicular shock by which specular reflection direction is nearly perpendicular to the magnetic field; (b) quasi-perpendicular shock by which specular reflection direction is oblique or parallel to the magnetic field (foreshock configuration); and (c) quasiparallel shock. We hereafter abbreviate these three types as QT, FS, and QL, respectively. A point that belongs to the FS bow shock is normally $90^{\circ}$ away in the azimuthal $(Y-Z)$ direction from a point that belongs to the QT shock as illustrated in Fig. 3(d). The difference between the FS and the QT cases is important in considering the pitch angle of specularly reflected solar wind because one must consider observation in the Mar frame and not in the de HoffmanTeller frame. The difference in the reference frames is important in the flank region where angle between the bow shock normal and the sun direction is more than $45^{\circ}$.

Note that it is nearly impossible to find an ideal quasiparallel case with MEX that does not carry the magnetometer (Yamauchi et al., 2008). For all examined traversals, IMA's ion counts at energies more than $0.5 \mathrm{keV}$ that are observed outside the bow shock are manually classified into ions from the solar wind, pickup ions with zero initial velocity ("primary ring distribution" in ion spectrograms), and accelerated ions. Electron data (ELS) is also used to supplement the ion data for identifying the shock crossings.

\section{Data}

IMA and ELS are parts of the Analyzer of Space Plasma and Energetic Atoms (ASPERA-3) experiment on board MEX. IMA is a top hat instrument that combines an electrostatic energy analyzer with a magnetic mass analyzer. IMA measures ions in the energy range from $10 \mathrm{eV} / \mathrm{q}$ to $30 \mathrm{keV} / \mathrm{q}$ in 96 logarithmically scaled energy steps every 12 sec. IMA has a $4.6^{\circ} \times 360^{\circ}$ field of view, where the $360^{\circ}$ measurement plane is divided into 16 azimuthal sectors $(0$ 15 ), each $22.5^{\circ}$ wide. Out of 16 sectors, $\phi=0$ is strongly contaminated from the other sectors, and we do not use it for the present analyses. IMA has an extra electrostatic deflection system (or elevation analyzer) at its entrance, which scans from $-45^{\circ}$ to $+45^{\circ}$ (16 elevations) in $192 \mathrm{sec}$. Therefore, IMA energy-time spectrograms have a 192-sec cycle, within which the spectrogram is seen as an energy-polar angle spectrogram. The overall field-of-view is approximately $360^{\circ}(16$ sectors $=\phi) \times 90^{\circ}(16$ elevations $=\theta)$. ELS is a 
top hat instrument that measures electrons, and has a $4^{\circ} \times$ $360^{\circ}$ field of view, which is divided into 16 azimuthal sectors, each $22.5^{\circ}$ wide. ELS covers an energy range from 1 $\mathrm{eV}$ to $20 \mathrm{keV}$. In 2005, ELS measured from a plane which was parallel to the IMA $0^{\circ}$ elevation plane. For details of the IMA and ELS instruments, see Barabash et al. (2006), Fedorov et al. (2006), and Frahm et al. (2006).

The orientation of the interplanetary magnetic field (IMF) is estimated from the ring distribution of newly-born ions coming from the planetary exosphere using the same method as that described in Yamauchi et al. (2011): These primary ring distribution is used to obtain the local L-M-N right-handed Cartesian coordinates through the minimum variance method, where $\mathbf{N}$ is the estimated IMF orientation (Yamauchi et al., 2006). In addition, $L_{X}=0$ is imposed to fill the last degree of freedom in the minimum variance method (this method constrains five components out of the total six components of $\mathbf{L}$ and $\mathbf{M}$ ), and the average $V_{N}$ is assumed to be zero for the primary ring ions (Yamauchi et al., 2011). This method is useful only when the IMF orientation is optimum $\left(B_{Y}^{2}+B_{Z}^{2}>B_{X}^{2}\right)$ because of the restrictive field of view of IMA due to the restrictive MEX attitude (driven by the camera which always faces the nadir direction).

\section{Observation}

Examination of the entire set of IMA quick-look spectrograms covering data from the first two years of observation (2004-2005) revealed six bow shock traversals that have clear and stable energy-bunched structures at discrete energies higher than that of the primary ring distribution at wide elevation (scanning) angles. All of them are found within $1000 \mathrm{~km}$ ( gyroradius of $2 \mathrm{keV}$ proton in $6 \mathrm{nT}$ magnetic field) from the bow shock. Note that this criterion might remove some ideal cases in which the ring ions are observed over several azimuthal sectors but not in the elevation direction (see example described in Yamauchi et al., 2008). We have examined these six traversals and the pass of 3 June 2005, for which the magnetic field orientation was estimated (Yamauchi et al., 2008) although the multiple bandlike structure was not obvious. Table 1 summarizes these seven traversals. The last traversal in Table 1 (12 July 2005) was examined in Yamauchi et al. (2011) and is summarized in the introduction. The orbits of these traversals are drawn in Fig. 2.

The third column in Table 1 shows the time period when IMA observed multiple accelerations nearly continuously in the upstream of the bow shock. The spacecraft positions during these traversals are drawn by solid and dashed lines in Fig. 2. The solid lines shown in Fig. 2 correspond to the time when multiple energy-bunched structures are observed. The fourth column in Table 1 shows the solar wind speed $V_{\mathrm{SW}}$ that is also given at the bottom of each panel in Fig. 6 using light blue color. The fifth column in Table 1 shows the angle $\theta_{X . n}$ between the $X$ axis and the normal direction (n) of the empirical bow shock model (Vignes et al., 2000).

Figure 4 shows the overview of proton and electron data from the first five traversals (\#1 to \#5) in the same format as Fig. 1(a). The electron data show fairly steady solar wind conditions, with increasing average energy toward the bow
Table 1. Selected seven traversals.

\begin{tabular}{lrccc}
\hline$\#$ & \multicolumn{1}{c}{ date } & UT & $V_{\mathrm{SW}}$ & $\theta_{X . n}$ \\
\hline$\# 1$ & 29 July 2005 & 17:26 17:49 & $360 \mathrm{~km} / \mathrm{s}$ & $41^{\circ}$ \\
$\# 2$ & 3 Aug. 2005 & $08: 46 \sim 11: 59$ & $470 \mathrm{~km} / \mathrm{s}$ & $43^{\circ}$ \\
$\# 3$ & 5 Aug. 2005 & $08: 22 \sim 10: 35$ & $400 \mathrm{~km} / \mathrm{s}$ & $50^{\circ}$ \\
$\# 4$ & 22 Nov. 2005 & $16: 57 \sim 18: 29$ & $370 \mathrm{~km} / \mathrm{s}$ & $59^{\circ}$ \\
$\# 5$ & 29 Nov. 2005 & $16: 05 \sim 16: 33$ & $380 \mathrm{~km} / \mathrm{s}$ & $31^{\circ}$ \\
$\# 6$ & 3 June 2005 & $05: 59 \sim 06: 29$ & $600 \mathrm{~km} / \mathrm{s}$ & $46^{\circ}$ \\
$\# 7$ & 12 July 2005 & $11: 35 \sim 11: 50$ & $350 \mathrm{~km} / \mathrm{s}$ & $56^{\circ}$ \\
\hline
\end{tabular}

shock at very close distance. The ion data shows several discrete bands at higher energy from that of the solar wind protons. These discrete bands do not correlate with any features in the electron data except during the bow shock crossing. Ions with the lowest band in energy and with the longest stretch in the elevation direction (the primary ring distribution) represent pick-up ions of exospheric hydrogen (Yamauchi et al., 2006, 2011), from which the orientation of the local magnetic field is estimated.

Using the same procedure as Yamauchi et al. (2011), the local L-M-N coordinate system was obtained for each full scan $(192 \mathrm{sec})$, and the velocity space points that registered five counts or more during the $100 \mathrm{~ms}$ measurement period are drawn in the $V_{L^{-}} V_{N}$ projection (upper panels) and $V_{L^{-}}$ $V_{M}$ projection (lower panels) for eight selected full scans from these many scans (other 192-sec scans are similar or less prominent) in Fig. 5 in the same format as Fig. 1(c). The corresponding energy versus elevation/time spectrograms that have been divided into different azimuthal sectors are shown in Fig. 6 (same format as Fig. 1(b)) such that one can relate the classification to the raw data.

We need both Figs. 5 and 6 in the analyses because IMA does not cover all $4 \pi$ direction and some of its field-ofview is blocked by the spacecraft (Yamauchi et al., 2011). The non-visible direction lies mostly at the lower part of the $V_{L}-V_{M}$ plots. Different energy-bunched stripes in Fig. 6 are classified using different colors in the similar way as Yamauchi et al. (2011), and these colors are used in Fig. 5: solar wind by light blue, the primary ring distribution by blue, the second ring distribution at energy higher than the primary ring distribution by orange, the third ring distribution at energy higher than the second ring distribution by red, and the other major clusters of counts by purple and green. The pink color is used for possible extension but disconnected from the red-colored branch.

The estimated IMF orientation (the $\mathbf{N}$ direction) is given at the top of each panel in Fig. 5, and is summarized in Table 2 (third column) for these selected scans shown in Fig. 5. The modeled bow shock normal n (cf. Table 1) in the L-M-N coordinates is drawn in Fig. 5 and given in Table 2 (fourth column). The types of bow shock in terms of the classification shown in Fig. 3 (QT, FS, and QL) are estimated from $\mathbf{n}$ near the MEX location and $\mathbf{N}$, and are summarized in the fifth column in Table 2 (also in the second column of Table 3 ). The sixth column in Table 2 shows the angle $\theta_{B . n}$ between the estimated IMF direction and $\mathbf{n}$. Due to substantial change in the IMF orientation during two traversals (3 August 2005 and 3 June 2005), the 


\section{MEX/ASPERA-3 (ELS \& IMA)}
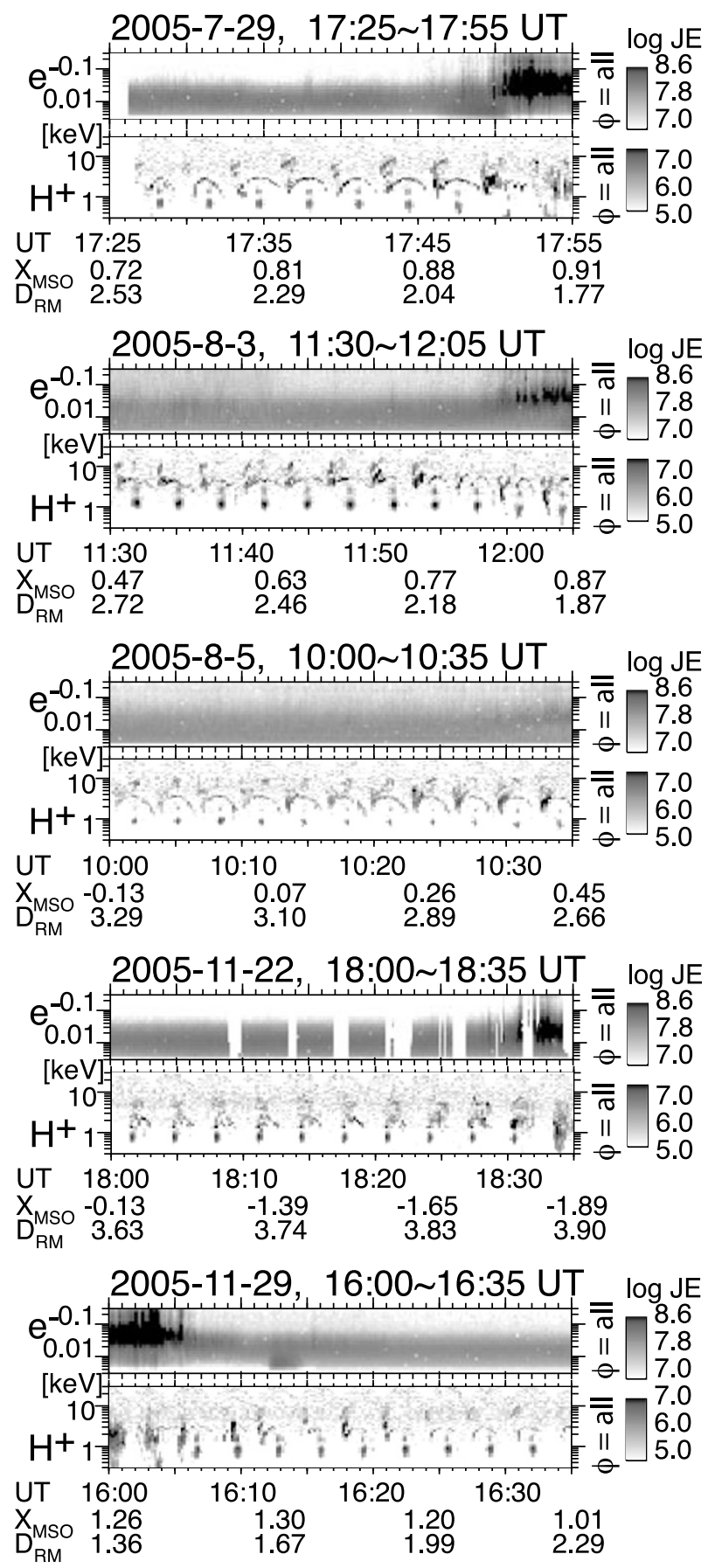

Fig. 4. Energy-time spectrograms of the energy flux $\left(\mathrm{keV} \mathrm{cm} \mathrm{cm}^{-2} \mathrm{~s}^{-1}\right.$ $\mathrm{keV}^{-1}$ ) of electrons and protons during five different traversals that are listed in Table 1 (\#1 to \#5). The format is the same as Fig. 1(a).

type of bow shock changed within $10 \mathrm{~min}$.

By studying the 12 July 2005 event, Yamauchi et al. (2011) concluded that the second ring distribution is specularly reflected solar wind at the bow shock (cf. Fig. 1(c)). To examine this for the present examples, we calculated the velocities of reflected solar wind from the bow shocks with normal direction $\mathbf{n}$ by assuming a specular reflection given by Eq. (1). The seventh and eighth columns in Table 2 show the estimated velocity and its error range (normalized by the solar wind speed) after the reflection in the $\mathbf{L}-\mathbf{M}-\mathbf{N}$ coordinates, respectively. The error range is calculated by assuming $\pm 5^{\circ}$ of uncertainty in the direction of $\mathbf{n}$ in both $X$ and $Y$ directions (the same as Yamauchi et al. (2011)). The calculated reflections are drawn using orange arrows in Fig. 5. The start and end of each arrow correspond to injection (solar wind) and reflection velocities, respectively. Due to the gyromotion of ions around the local magnetic field ( $V_{N}$ axis) in the solar wind frame, a reflected solar wind ion follows a circular trajectory in the $V_{L}-V_{M}$ plane as drawn using orange dashed circle while keeping a constant $V_{N}$ that is given by orange horizontal lines in Fig. 5 (the same as Fig. 1(c)). The observed secondary ring distribution (orange empty triangles) is found near such predicted trajectories.

Yamauchi et al. (2011) further showed that the third ring distribution at energy higher than the second ring distribution in the 12 July 2005 event agrees with second reflection of the reflected solar wind ions at the bow shock, as shown in Fig. 1(c). In this example, the clustered ions are found only within a short extension in the elevation direction, making a gyrophase bunched distribution as one can see in Figs. 1(a) and 1(b). The velocity that corresponds to the disappearance of the secondary ring distribution has a large anti-sunward speed (larger than the solar wind speed) and flowing toward the bow shock with normal $\mathbf{n}$ as one can see in Fig. 1(c). Therefore, the gyrophase that corresponds to the second injection to the bow shock (first injection is the solar wind) was assumed to be about the same as where the cluster of secondary ring distribution disappears.

The same type of gyrophase bunching and the large antisunward speed at the end of this bunching are observed in all cases in Figs. 4-6. Therefore, we assume the same, i.e., that the observed gyrophase extent (disappearance) of the second ring corresponds to the lost due to the second reflection. The velocities of the assumed second injection normalized by the solar wind speed in the $\mathbf{L}-\mathbf{M}-\mathbf{N}$ coordinates are shown in the fourth column of Table 3. The corresponding gyrophase angles in the solar wind frame are also shown in the fifth column. Since the ring distribution is aligned to the elevation direction (angular resolution is less than $10^{\circ}$ ) as shown in Fig. 4, the error range of the terminating gyrophase is about $\pm 10^{\circ}$, while we assumed the same shock normal $\mathbf{n}$ between the first and second reflections because most of the observations are where the bow shock curvature is small compared to the reflected solar wind. This procedure automatically assumes the direction of the gyration, which indicates the sign of the magnetic field polarity (sign of the fifth column in Table 3, where plus means clockwise rotation and minus means counter-clockwise rotation in Fig. 5).

Using these injection velocities, velocities of second specular reflections are calculated. The velocity and their error range in the L-M-N coordinates (normalized by the solar wind speed) are summarized in the sixth and seventh columns of Table 3. A secondary reflected ion again performs gyromotion around the IMF ( $V_{N}$ axis) in the solar wind frame, and it follows a circular trajectory in the $V_{L^{-}}$ $V_{M}$ plane while keeping a constant $V_{N}$. The jump of the velocity by this reflection in the $V_{L}-V_{M}$ projection is also 

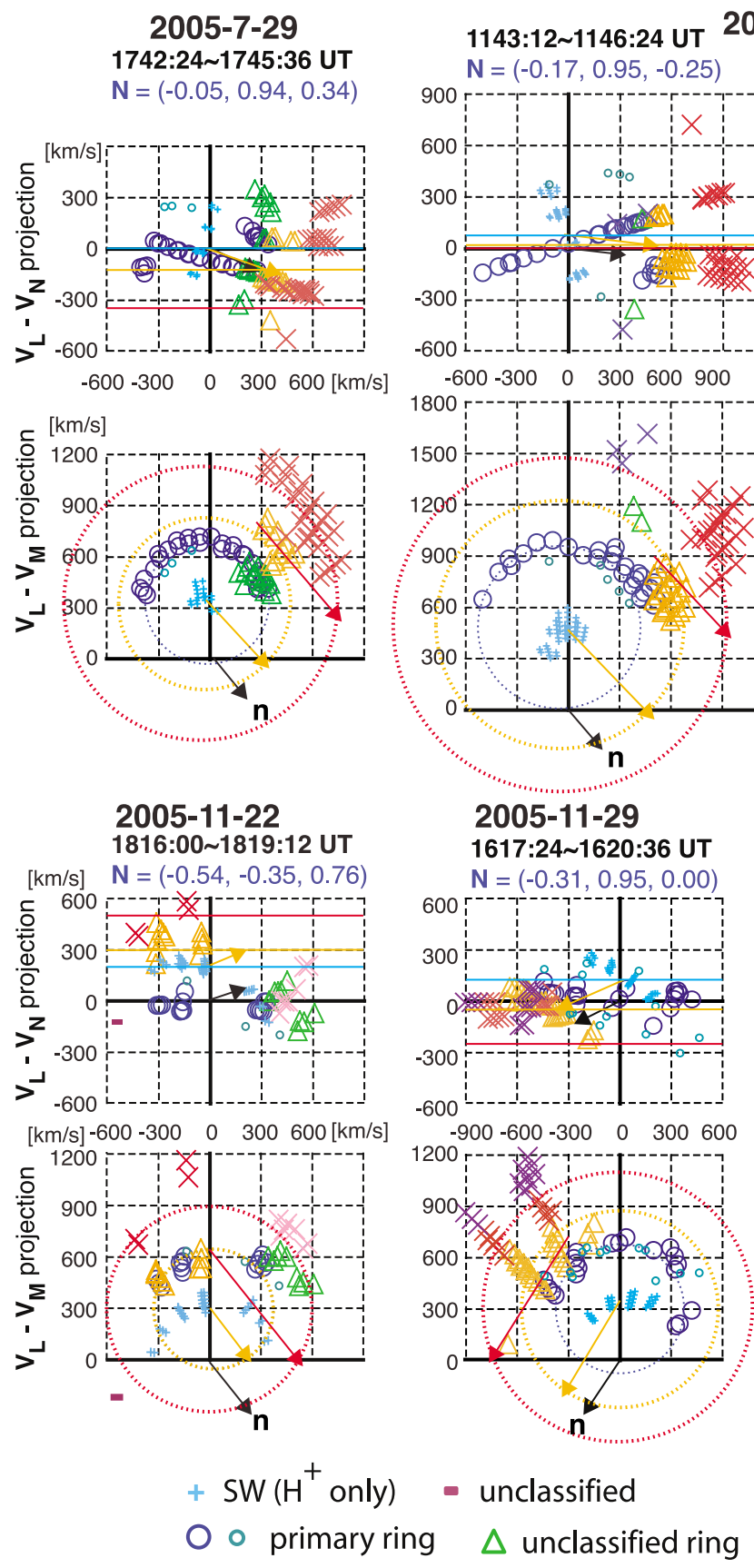

MEX/IMA, $\mathrm{H}^{+}$data 1143:12 1146:24 UT 2005-8-3 1152:48 1156:00 UT $\mathbf{N}=(-0.17,0.95,-0.25)$
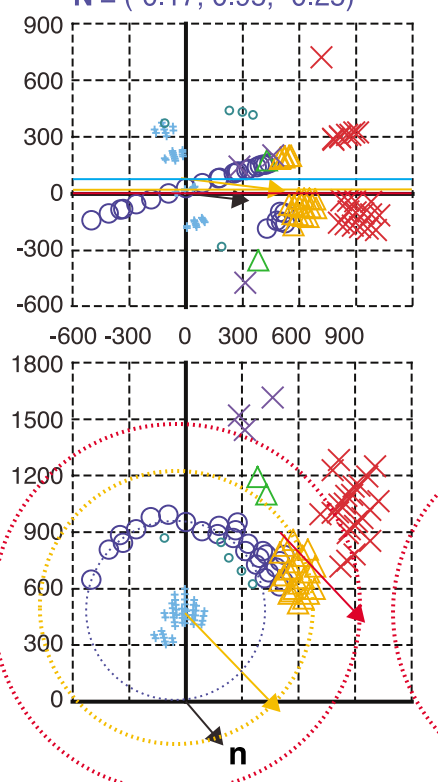

1617:24 1620:36 UT

$\Delta$ unclassified ring

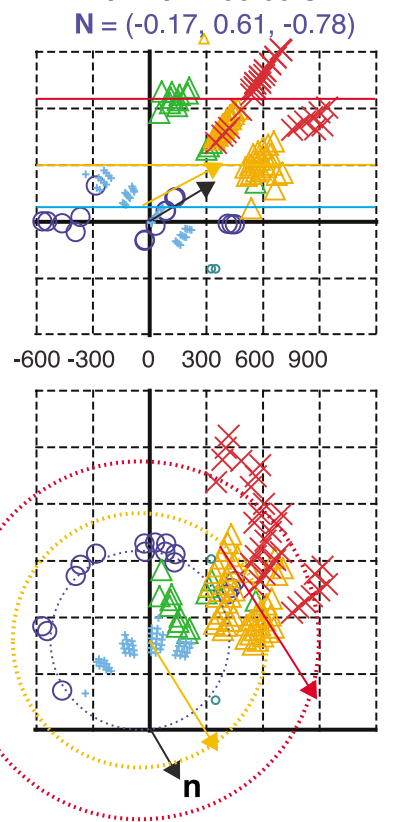

2005-8-5

1000:12 1003:24 UT

$\mathrm{N}=(-0.07,0.82,0.57)$

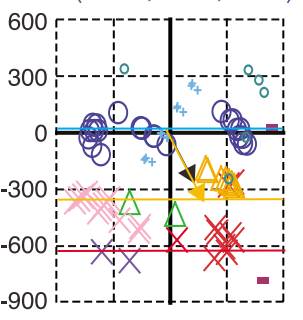

$-600-300 \quad 0 \quad 300600$

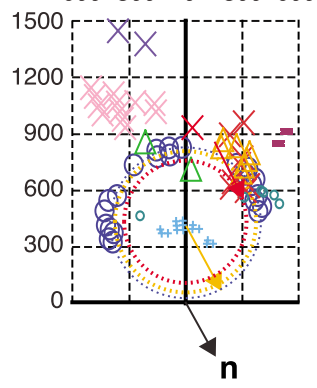

2005-6-3

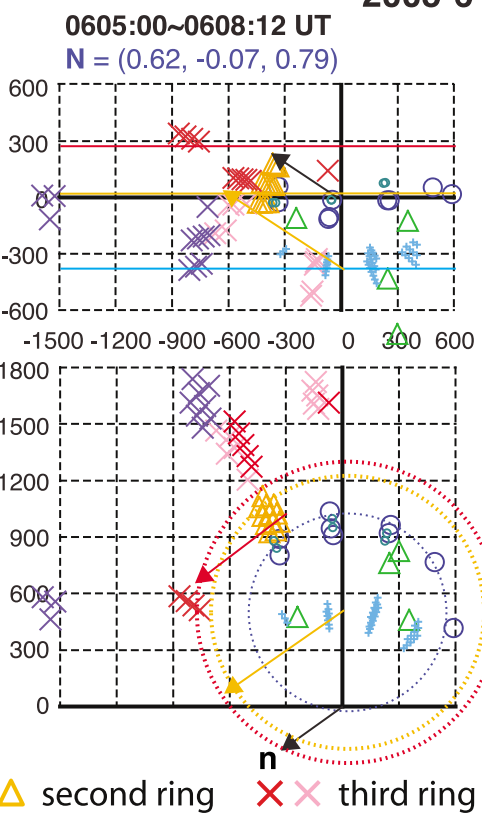

0611:24 0614:36 UT

$\mathbf{N}=(0.57,-0.56,0.60)$

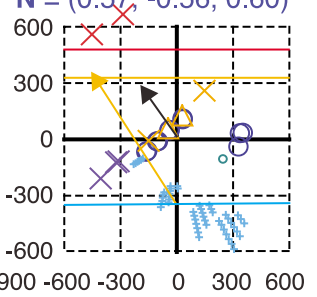

$-900-600-300 \quad 0 \quad 300600$

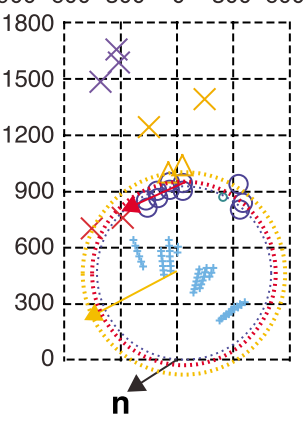

\section{$X$ highest ring}

Fig. 5. Velocity scatter plots of selected scans (192 sec data each) from traversals shown in Fig. 2 (taking periods given in Table 2). The format is the same as Fig. 1(c). The same colors are used in Fig. 6.

drawn by red arrows in Fig. 5, and the following gyromotion is drawn by a red dashed circle. The same prediction is also made in the field-aligned direction, as given by the red horizontal lines in the $V_{L}-V_{N}$ projection. The observed third ring (red cross) in Fig. 5 is found at velocity space near this prediction for all cases, and therefore, the third ring distribution is well explained by the secondary specular reflection of the solar wind ions.

Note that possible extensions of the third ring that are marked by pink colors for 5 Aug 2005 event (\#3a) and 3 June 2005 events (\#6a, \#6b) are slightly off from the expected gyrating trajectory. This means that the pink-marked ions and red-marked ions are not on the same trajectory in velocity space. Since the bow shock normal direction is not constant in both time and space, such a fluctuation is quite possible, constructing a deformed ring trajectory. In fact, the magnetic field direction changed dynamically during the traversal of 3 June, 2005 (Yamauchi et al., 2008).

Figure 5 shows another clustered population marked by green triangles. This population extends from velocity space point that is very close to the primary ring distribution, whereas it does not resemble to the foot ions that are studied in Yamauchi et al. (2011). We have no solid interpretation for this population, and can only speculate that 


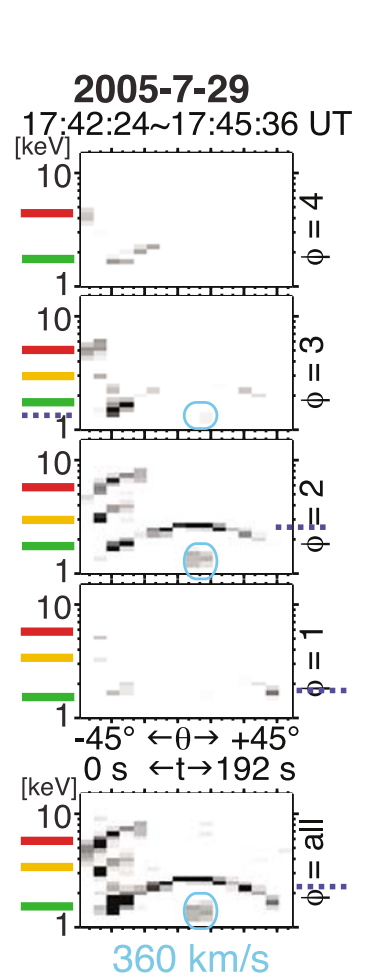

\section{MEX/IMA, $\mathrm{H}^{+}$data} 2005-8-3
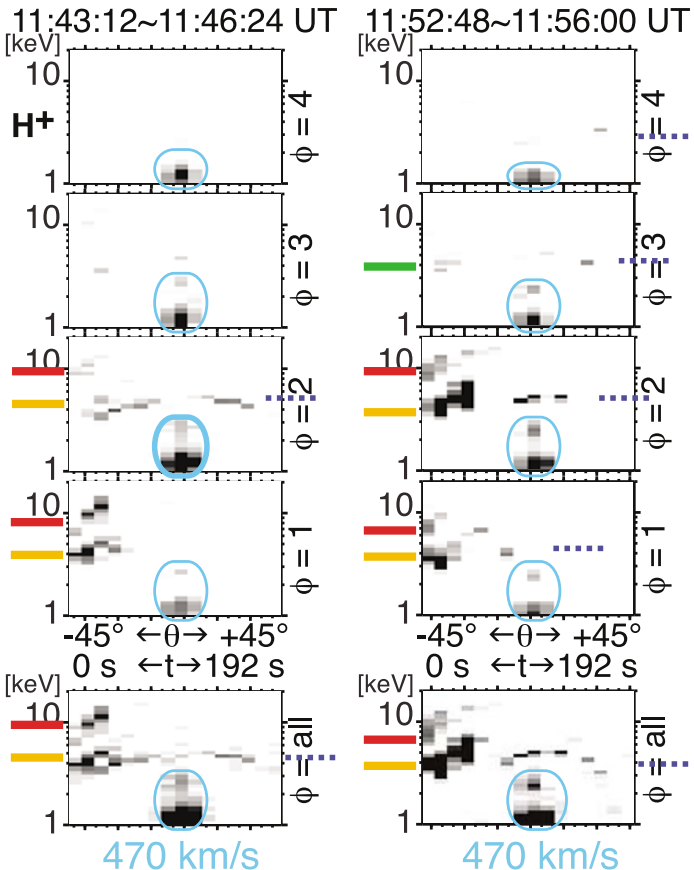

2005-8-5
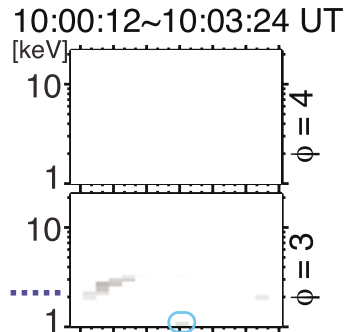

2005-11-22

2005-11-29
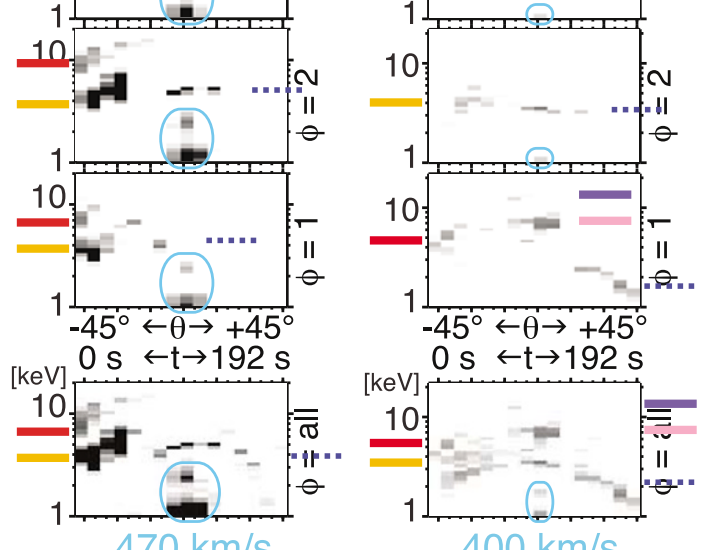

8:16:00 18:19:12 UT 16:17:24 16:20
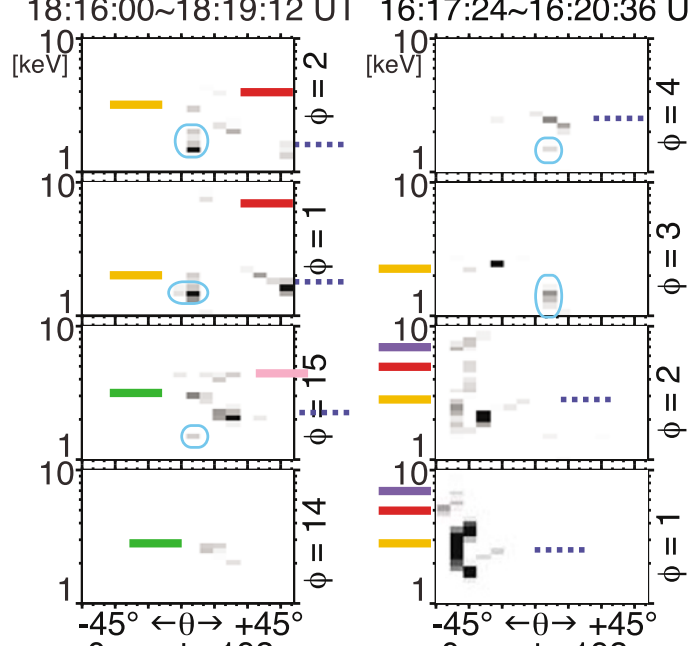

2005-6-3

$400 \mathrm{~km} / \mathrm{s}$

:12 UT 06:11:24 06:14:36 UT
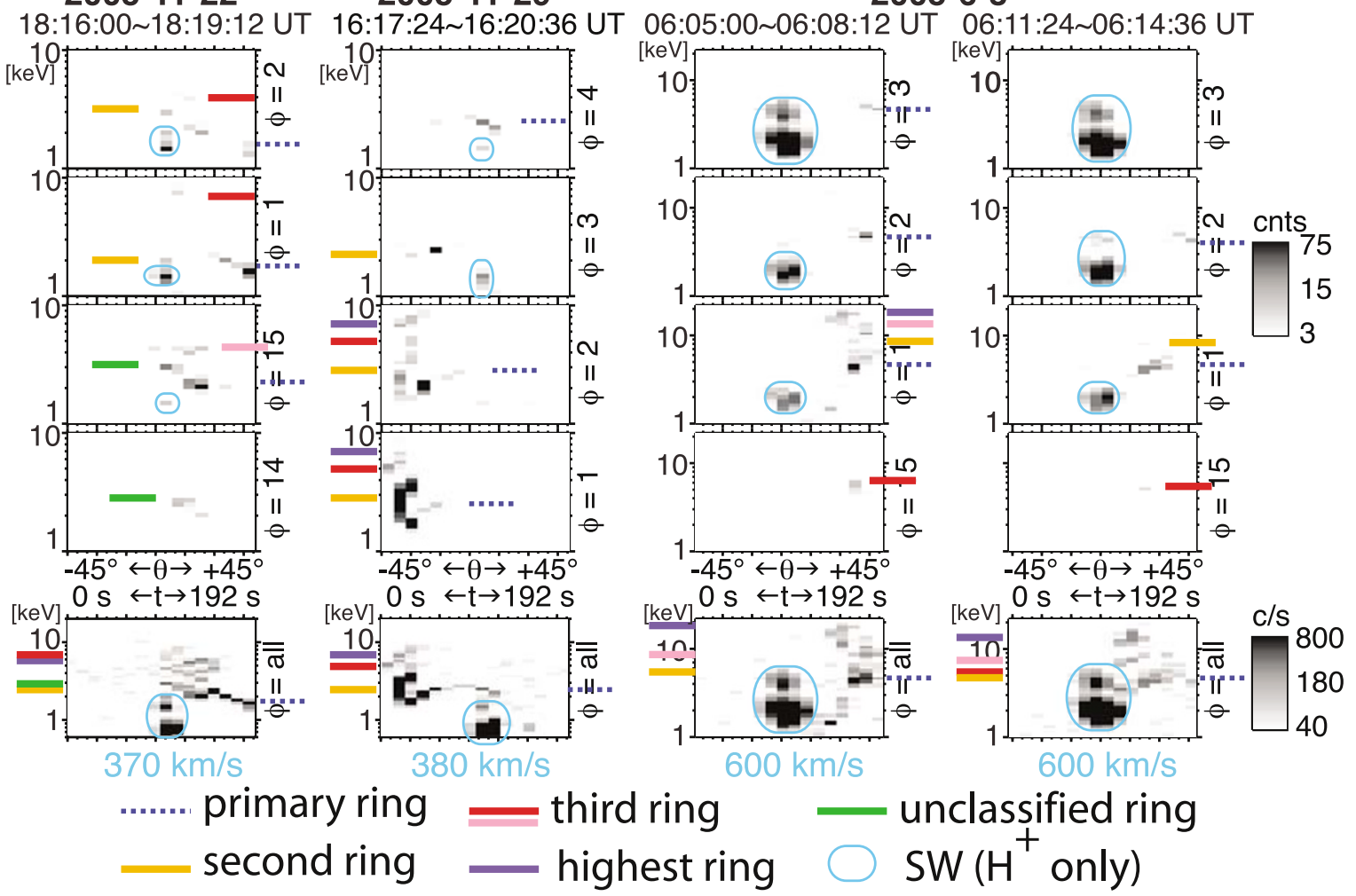

Fig. 6. Energy-time spectrograms from MEX/IMA proton channels for full-scan (192 s) data that correspond to Fig. 5. The format is the same as Fig. 1(b). Sector 0 is not presented because it gathers contamination from all of the other sectors. The same colors are used in Fig. 5 . Note that the solar wind $\mathrm{He}^{++}$contaminates at energy twice the solar wind proton energy.

it might be related to the interaction between the pick-up ions and the bow shock. Note that pick-up ions can enter the bow shock at wide gyro-phases, and cannot introduce the narrow reflection direction that is necessary for energybunched second and third rings.

\section{Summary and Discussion}

We have examined seven different examples of which six are the best traversals during 2005 of multiple energybunched protons at energies higher than the pickup ions of exospheric origin (zero-velocity in the planetary rest 
Table 2. Selected seven traversals.

\begin{tabular}{|c|c|c|c|c|c|c|c|}
\hline$\#$ & UT & $\mathbf{N}$ in $\mathrm{MSO}$ & $\mathbf{n}$ in $\mathrm{LMN}$ & type & $\theta_{B . n}$ & $\mathbf{V}_{R 1} / V_{\mathrm{SW}}$ in $\mathrm{LMN}$ & error \\
\hline$\# 1 \mathrm{a}$ & $17: 43$ & $(-0.05,+0.94,+0.34)$ & $(+0.62,-0.74,-0.26)$ & QT & $-75^{\circ}$ & $(+0.93,-0.12,-0.35)$ & $\pm(0.04,0.17,0.01)$ \\
\hline$\# 2 \mathrm{a}$ & $11: 46$ & $(+0.05,+0.95,-0.25)$ & $(+0.68,-0.73,-0.07)$ & QT & $-86^{\circ}$ & $(+0.99,-0.08,+0.07)$ & $\pm(0.02,0.17,0.03)$ \\
\hline$\# 2 b$ & $11: 53$ & $(-0.17,+0.61,-0.78)$ & $(+0.51,-0.80,+0.32)$ & FS & $+71^{\circ}$ & $(+0.74,-0.18,+0.65)$ & $\pm(0.02,0.17,0.05)$ \\
\hline$\# 3 \mathrm{a}$ & 10:01 & $(-0.07,+0.79,+0.61)$ & $(+0.32,-0.59,-0.74)$ & QL & $-42^{\circ}$ & $(+0.42,+0.24,-0.88)$ & $\pm(0.02,0.18,0.05)$ \\
\hline$\# 4 \mathrm{a}$ & $18: 17$ & $(-0.54,-0.35,+0.76)$ & $(+0.61,-0.76,+0.23)$ & QT & $+77^{\circ}$ & $(+0.62,+0.05,+0.78)$ & $\pm(0.06,0.16,0.05)$ \\
\hline$\# 5 \mathrm{a}$ & $16: 17$ & $(-0.31,+0.95,0.00)$ & $(-0.52,-0.82,-0.26)$ & QT & $-75^{\circ}$ & $(-0.88,-0.45,-0.15)$ & $\pm(0.09,0.16,0.05)$ \\
\hline$\# 6 \mathrm{a}$ & 06:06 & $(+0.62,-0.07,+0.79)$ & $(-0.72,-0.51,+0.48)$ & QT & $+62^{\circ}$ & $(-1.00,+0.08,+0.05)$ & $\pm(0.03,0.14,0.11)$ \\
\hline$\# 6 \mathrm{~b}$ & $06: 13$ & $(+0.57,-0.56,+0.60)$ & $(-0.53,-0.29,+0.80)$ & QL & $+37^{\circ}$ & $(-0.73,+0.42,+0.54)$ & $\pm(0.02,0.15,0.11)$ \\
\hline$\# 7 \mathrm{a}$ & $11: 47$ & $(+0.26,+0.91,+0.33)$ & $(-0.28,-0.74,-0.61)$ & FS & $-53^{\circ}$ & $(-0.32,+0.14,-0.94)$ & $\pm(0.03,0.18,0.03)$ \\
\hline
\end{tabular}

Table 3. Selected seven traversals.

\begin{tabular}{|c|c|c|c|c|c|c|c|c|c|c|}
\hline$\#$ & type & $\theta_{B . n}$ & $\mathbf{V}_{I 2} / V_{\mathrm{SW}}$ & gyro & $\mathbf{V}_{R 2} / V_{\mathrm{SW}}$ & error & accel. & $\mathbf{V}_{\mathrm{dHT}} / V_{\mathrm{SW}}$ & error $_{\|}$ & escape \\
\hline$\# 1 \mathrm{a}$ & QT & $-75^{\circ}$ & $(+0.8,+2.2,-0.4)$ & $-110^{\circ}$ & $(+2.1,+0.7,-0.9)$ & $\pm(0.2,0.1,0.2)$ & $\perp$ & $(0,1.0,-2.8)$ & \pm 0.8 & no \\
\hline$\# 2 \mathrm{a}$ & QT & $-86^{\circ}$ & $(+1.1,+1.9,+0.1)$ & $-90^{\circ}$ & $(+2.0,+1.0,0.0)$ & $\pm(0.2,0.2,0.1)$ & $\perp$ & $(0,1.0,-11)$ & $\infty$ & no \\
\hline$\# 2 b$ & FS & $+71^{\circ}$ & $(+0.8,+2.1,+0.7)$ & $-110^{\circ}$ & $(+1.9,+0.4,+1.4)$ & $\pm(0.1,0.2,0.2)$ & obl. & $(0,1.0,2.4)$ & \pm 0.7 & no \\
\hline$\# 3 \mathrm{a}$ & QL & $-42^{\circ}$ & $(+0.4,+2.1,-0.9)$ & $-130^{\circ}$ & $(+0.7,+1.6,-1.6)$ & $\pm(0.1,0.1,0.2)$ & $\|$ & $(0,1.0,-0.8)$ & \pm 0.2 & yes \\
\hline$\# 4 \mathrm{a}$ & QT & $+77^{\circ}$ & $(0.0,+1.8,+0.8)$ & $+220^{\circ}$ & $(+1.4,0.0,+1.3)$ & $\pm(0.1,0.2,0.1)$ & obl. & $(0,0.8,2.8)$ & \pm 1.4 & no \\
\hline$\# 5 \mathrm{a}$ & QT & $-75^{\circ}$ & $(-0.8,+1.9,-0.1)$ & $+120^{\circ}$ & $(-1.9,+0.1,-0.7)$ & $\pm(0.1,0.2,0.2)$ & $\perp$ & $(0,1.0,-2.9)$ & \pm 0.6 & no \\
\hline$\# 6 \mathrm{a}$ & QT & $+62^{\circ}$ & $(-0.5,+1.7,+0.1)$ & $+100^{\circ}$ & $(-1.2,+1.2,+0.5)$ & $\pm(0.2,0.1,0.2)$ & $\perp$ & $(0,0.8,0.8)$ & \pm 0.2 & no \\
\hline$\# 6 b$ & $\mathrm{QL}$ & $+37^{\circ}$ & $(+0.1,+1.6,+0.1)$ & $+130^{\circ}$ & $(-0.4,+1.4,+0.8)$ & $\pm(0.1,0.2,0.1)$ & $/ /, \perp$ & $(0,0.8,0.3)$ & \pm 0.1 & yes \\
\hline$\# 7 \mathrm{a}$ & FS & $-53^{\circ}$ & $(-0.3,+2.5,-1.0)$ & $-210^{\circ}$ & $(-1.0,+0.8,-2.4)$ & $\pm(0.2,0.1,0.1)$ & $\|$ & $(0,1.0,-1.2)$ & \pm 0.2 & yes \\
\hline
\end{tabular}

frame). In all cases that are summarized in Tables 1 and 2, the second branch marked by orange colors in Figs. 5 and 6 are consistent with specular reflections of the solar wind at the bow shock in the Mars frame (Sagdeev, 1966; Gosling et al., 1978; Paschmann et al., 1980, 1981; Dubinin et al., 2006; Yamauchi et al., 2011); i.e., both parallel velocity and perpendicular speed in the solar wind frame (with respect to the magnetic field) of the specularly reflected solar wind agrees with those of the energy-bunched ions marked by the orange color. A large part of the data is further consistent with second reflection of these orange-marked ions, although some traversals have ion populations that do not belong to this explanation.

We also examined two observations from the 3 June 2005 traversal when the IMF changed quickly. The first observation at around 06:07 UT (\#6a) showed energy-bunching that is consistent with the multiple specular reflections at the bow shock. The other observation at around 06:13 UT (\#6b) showed weak ion counts that is consistent with the multiple reflection and a substantial counts of accelerated ions that does not agree with the reflection prediction. However, the latter is somewhat similar to \#6a with less intensity, and might be remaining from the old population because a temporal variation in the IMF can easily cause a reconfiguration of the bow shock or even non-planer bow shock. The energized ions arriving at the IMA instrument during 06:11:24 06:14:36 UT might have come from a different region than the pick-up ions.

Except \#6b mentioned above, the predicted reflection direction with respect to the estimated magnetic field orientation agrees with the observed energy-bunched stripes, and this direction is summarized in the eighth column in Table 3 (observation is shown for \#6b). Perpendicular accelerations (\#1a, \#2a, \#5a, \#6a) are consistently seen in the QT shocks, whereas parallel accelerations (\#3a, \#6b, \#7a) are seen in the QL and FS shocks. In this sense, the sub-classification of the quasi-perpendicular shock into QT and FS turned out to be useful. The other cases (\#2b, \#4a) are marginal and consistent with the above relation. These relations agree with the terrestrial bow shock where foreshock ions are found upstream of the quasi-parallel shocks (e.g., Eastwood et al., 2005; and references therein).

The finite field-aligned velocity indicates that these ions may escape depending on the bow shock configuration with respect to the magnetic field. To diagnosis this, we estimated the de Hoffman-Teller velocity

$$
\mathbf{V}_{\mathrm{dHT}}=\mathbf{n} \times\left(\mathbf{V}_{\mathrm{SW}} \times \mathbf{N}\right) /(\mathbf{N} \cdot \mathbf{n})
$$

from the solar wind velocity $\mathbf{V}_{\mathrm{SW}}$, the estimated IMF orientation $\pm \mathbf{N}$ (sign disappears from $\mathbf{V}_{\mathrm{dHT}}$ ) and the modeled bow shock normal $\mathbf{n}$. The escape condition is determined by the sign of $\left(V_{N}-V_{\mathrm{dHT}_{N}}\right) \cdot n_{N}$ (escape corresponds to positive), while we cannot simply use $\mathbf{V}_{\mathrm{dHT}}$ in judging the actual escape because finite gyroradius makes ions easier to return back and because the de Hoffman-Teller analyses can be erroneous at the flank bow shock (Yamauchi et al., 2011). With these limitation in mind, de Hoffman-Teller analyses provide some guide.

The ninth and tenth columns of Table 3 summarize $\mathbf{V}_{\mathrm{dHT}}$ and its error range in the field-aligned direction by assuming $\pm 5^{\circ}$ uncertainty in the direction of $\mathbf{n}$. Except for 3 June 2005 events (\#6a, \#6b) and 5 August 2005 event (\#3a), $\left|\mathbf{V}_{\mathrm{dHT}}\right| / V_{\mathrm{SW}}>1.5$. Furthermore, the error in the fieldaligned component of $\mathbf{V}_{\mathrm{dHT}}$ is larger than half the solar wind speed in 5 examples. Therefore, our estimate of the escape condition in the presented examples has large uncertainty.

On the other hand, all events with parallel acceleration (\#3a, \#6b, \#7a) correspond to escape beyond the uncer- 
tainty after the second reflection, as summarized in eleventh column of Table 3 . The velocities after the first reflection in these events are marginal (between escape and being trapped), and the finite gyroradius of ions cause them to hit the bow shock again (Yamauchi et al., 2011). The events with perpendicular acceleration correspond to trapping, although the uncertainty is large for these cases. From these results, escape from the bow shock seems to require a configuration in which ions are reflected toward the fieldaligned direction such as at the QL shocks.

In the present study, we have examined only cases when multiple accelerations (energy-bunched structures) are observed. We did not examine general cases when such structure is not observed such as the case of 3 June 2005 (\#6a, \#6b). The latter study is needed to know the probability of observing the energy-bunched structures. Unfortunately, such a statistical study is difficult, if not impossible, without a magnetometer. We leave it to the future studies from a new Martian mission with a full plasma-field package.

In all examples that are ideal for analyses during 2005, the intense counts at higher energy than the primary ring distribution are often found immediately outside the Martian bow shock, and these intense counts at energies of several to tens keV are most likely accelerated solar wind ions by multiple specular reflections at the bow shock. Although the present study is not statistically strong, the data support common occurrence of multiple specular reflection of the solar wind at the bow shock, and hence support the explanation by the abundance of cold ions at the Martian bow shock as the reason for the speciality of Mars, i.e., not reported at the Earth or Venus (Yamauchi et al., 2011).

Let us consider the possible consequence if the cold ambient ions caused the solar wind acceleration by multiple reflections at the bow shock. Combination of the cold ions and the shocks is expected in the interstellar medium because it is rich in cold neutrals. For example, shocks associated with supernova explosions may have abundant cold ions. Since the multiple reflection mechanism is very effective for bulk acceleration, it may well pre-accelerate ions at the supernova remnant shock before the Fermi acceleration takes over to create galactic cosmic rays. A similar mechanism has been simulated for electrons (Amano and Hoshino, 2009), and we need similar simulation for ions in the future.

Acknowledgments. This work was partly supported by the Swedish Research Counsel (VR) \#621-2008-3769 and Swedish National Space Board (RS) in Sweden, and by NASA contract NASW-00003 at SwRI, in the USA. The lead author (MY) wishes to thank Sweden programs of providing help for physically disabled individuals which have made it possible for him to conduct this work.

Guest editor J. S. Halekas thanks two anonymous reviewers for their assistance in evaluating this paper.

\section{References}

Afonin, V., S. McKenna-Lawlor, K. Gringauz, K. Kecskemety, E. Keppler, E. Kirsch, A. Richter, D. O'Sullivan, A. Somogyi, A. Thompson, A. Varga, and M. Witte, Energetic ions in the close environment of Mars and particle shadowing by the planet, Nature, 341, 616-618, doi:10.1038/341616a0, 1989.

Amano, T. and M. Hoshino, Electron shock surfing acceleration in multidimensions: Two-dimensional particle-in-cell simulation of collisionless perpendicular shock, Astrophys. J., 690, 244-251, doi:10.1088/0004637X/690/1/244, 2009.

Bale, S. D., M. A. Balikhin, T. S. Horbury, V. V. Krasnoselskikh, H. Kucharek, E. Möbius, S. N. Walker et al., Quasi-perpendicular shock structure and processes, Space Sci. Rev., 118, 161-203, doi:10.1007/s11214-005-3827-0, 2005.

Barabash, S., R. Lundin, H. Andersson, K. Brinkfeldt, A. Grigoriev, H. Gunell et al., The analyzer of space plasmas and energetic atoms (ASPERA-3) for the Mars Express mission, Space Sci. Rev., 126(1-4), 113-164, doi:10.1007/s11214-006-9124-8, 2006.

Burgess, D. and S. J. Schwartz, The Dynamics and upstream distributions of ions reflected at the Earth's bow shock, J. Geophys. Res., 89(A9), 7407-7422, doi:10.1029/JA089iA09p0740, 1984.

Burgess, D., E. A. Lucek, M. Scholer, S. D. Bale, M. A. Balikhin, A Balogh, T. S. Horbury, V. V. Krasnoselskikh, H. Kucharek, B. Lembege, E. Möbius, S. J. Schwartz, M. F. Thomsen, and S. N. Walker, Quasiparallel shock structure and processes, Space Sci. Rev., 118, 205-222, doi:10.1007/s11214-005-3827-0, 2005.

Dubinin, E., R. Lundin, H. Koskinen, and O. Norberg, Cold ions at the Martian bow shock phobos observations, J. Geophys. Res., 98, 56175623, 1993

Dubinin, E., M. Fraenz, J. Woch, S. Barabash, R. Lundin, and M Yamauchi, Hydrogen exosphere at Mars: Pickup protons and their acceleration at the bow shock, Geophys. Res. Lett., 33, L22103, doi:10.1029/2006GL027799, 2006.

Eastwood, J. P., E. A. Lucek, C. Mazelle, K. Meziane, Y. Narita, J. Pickett, and R. A. Treumann, The foreshock, Space Sci. Rev., 118, 41-94, doi:10.1007/s11214-005-3824-3, 2005.

Fedorov, A., E. Budnik, J.-A. Sauvaud, C. Mazelle, S. Barabash, R. Lundin et al., Structure of the Martian wake, Icarus, 182(2), 329-336, doi:10.1016/j.icarus.2005.09.021, 2006.

Frahm, R. A., J. D. Winningham, J. R. Sharber, J. R. Scherrer, S. J. Jeffers, A. J. Coates, D. R. Linder, D. O. Kataria, R. Lundin, and S. Barabash et al., Carbon dioxide photoelectron energy peaks at Mars, Icarus, 182, 371-382, 2006

Gloeckler, G., J. Geiss, E. C. Roelof, L. A. Fisk, F. M. Ipavich, K. W. Ogilvie, L. J. Lanzerotti, R. von Steiger, and B. Wilken, Acceleration of Interstellar Pickup Ions in the Disturbed Solar Wind Observed on Ulysses, J. Geophys. Res., 99(A9), 17637-17643, 1994.

Gosling, J. T., J. R. Asbridge, S. J. Bame, G. Paschmann, and N. Sckopke, Observations of two distinct populations of bow shock ions in the upstream solar wind, Geophys. Res. Lett., 5(11), 957-960, 1978.

Gosling, J. T., M. F. Thomsen, S. J. Bame, W. C. Feldman, G. Paschmann, and N. Sckopke, Evidence for specularly reflected ions upstream from the quasi-parallel bow shock, Geophys. Res. Lett., 9(12), 1333-1336, 1982.

Lee, M. A., V. D. Shapiro, and R. Z. Sagdeev, Pickup ion energization by shock surfing, J. Geophys. Res., 101(A3), 4777-4789, 1996.

Mazelle, C., K. Meziane, D. Le Queau, M. Wilber, J. P. Eastwood, H. Re, J. A. Sauvaud et al., Production of gyrating ions from nonlinear waveparticle interaction upstream from the Earth's bow shock: a case study from Cluster-CIS, Planet. Space Sci., 51, 785-795, 2003.

McKenna-Lawlor, S., V. V. Afonin, E. Kirsch, K. Schwingenschuh, J. A Slavin, and J. G. Trotignon, An overview of energetic particles (from $55 \mathrm{keV}$ to $>30 \mathrm{MeV}$ ) recorded in the close Martian environment, and their energization in local and external processes, Planet. Space Sci., 46, 83-102, 1998.

Meziane, K., C. Mazelle, R. P. Lin, D. LeQuéau, D. E. Larson, G. K Parks, and R. P. Lepping, Three-dimensional observations of gyrating ion distributions far upstream from the Earth's bow shock and their association with low-frequency waves, J. Geophys. Res., 106, 5731$5742,2001$.

Oka, M., T. Terasawa, Y. Saito, and T. Mukai, Field-aligned beam observations at the quasi-perpendicular bow shock: Generation and shock angle dependence, J. Geophys. Res., 110, A05101, doi:10.1029/2004JA010688, 2005.

Paschmann, G., N. Sckopke, J. R. Asbridge, S. J. Bame, and J. T. Gosling, Energization of solar wind ions by reflection from the Earth's bow shock, J. Geophys. Res., 85, 4689-4693, 1980.

Paschmann, G., N. Sckopke, I. Papamastorakis, J. R. Asbridge, S. J. Bame, and J. T. Gosling, Characteristics of reflected and diffuse ions upstream from the Earth's bow shock, J. Geophys. Res., 86, 4355-4364, 1981.

Sagdeev, R. Z., Cooperative phenomena and shock waves in collisionless plasmas, in Reviews of Plasma Physics, vol. 4, edited by M. A. Leontovich, p. 23, Consultants Bur., New York, 1966.

Sckopke, N., G. Paschmann, S. J. Bame, J. T. Gosling, and C. T. Rus- 
sell, Evolution of ion distributions across the nearly perpendicular bow shock: Specularly and non-specularly reflected-gyrating ions, J. Geophys. Res., 88, 6121-6136, 1983.

Sckopke, N., G. Paschmann, A. L. Brinca, C. W. Carlson, and H. Luhr, Ion thermalization in quasi-perpendicular shocks involving reflected ions, $J$. Geophys. Res., 95, 6337-6352, 1990.

Vignes, D., C. Mazelle, H. Reme, M. H. Acuna, J. E. P. Connerney, R. P. Lin, D. L. Mitchell, P. Cloutier, D. H. Crider, and N. F. Ness, The solar wind interaction with Mars: Locations and shapes of the bow shock and the magnetic pile-up boundary from the observations of the MAG/ER Experiment onboard Mars Global Surveyor, Geophys. Res. Lett., 27(1), 49-52, doi:10.1029/1999GL010703, 2000.

Yamauchi, M., Y. Futaana, A. Fedorov, E. Dubinin, R. Lundin, J.-A. Sauvaud, D. Winningham, R. Frahm, S. Barabash et al., IMF direction derivation from cycloid-like ion distributions observed by Mars Express, Space Sci. Rev., 126(1-4), 239-266, doi:10.1007/s11214-0069090-1, 2006.

Yamauchi, M., Y. Futaana, A. Fedorov, E. Kallio, R. A. Frahm, R. Lundin, J.-A. Sauvaud, D. J. Winningham, S. Barabash, and M. Holm- ström, Advanced method to derive the IMF direction near Mars from cycloidal proton distributions, Planet. Space Sci., 56(8), 1145-1154, doi:10.1016/j.pss.2008.02.012, 2008.

Yamauchi, M., Y. Futaana, E. Fedorov, R. A. Frahm, J. D. Winningham, E. Dubinin, R. Lundin, S. Barabash, M. Holmström, C. Mazelle, J.-A. Sauvaud, T. L. Zhang, W. Baumjohann, A. J. Coates, and M. Fraenz, Comparison of accelerated ion populations observed upstream of the bow shocks at Venus and Mars, Ann. Geophys., 29, 511-528, doi:10.5194/angeo-29-511-2011, 2011.

Zank, G. P., H. L. Pauls, I. H. Cairns, and G. M. Webb, Interstellar pickup ions and quasi-perpendicular shocks: Implications for the termination shock and interplanetary shocks, J. Geophys. Res., 101(A1), 457-477, 1996.

M. Yamauchi (e-mail: M.Yamauchi@irf.se), Y. Futaana, A. Fedorov, R. A. Frahm, E. Dubinin, R. Lundin, J.-A. Sauvaud, J. D. Winningham, S. Barabash, and M. Holmström 